\section{Begünstigt die Aknetherapie eine Pharyngitis?}

US-amerikanische Kollegen haben weitere Hinweise dafür gefunden, dass die Verwendung oraler Antibiotika zur Behandlung der Akne sehr eng mit der Entwicklung einer Pharyngitis assoziiert ist.

S eit mehr als fünf Jahren vermuten Forscher einen Zusammenhang zwischen Antibiotika gegen Akne und dem Auftreten von Pharyngitiden. In einer aktuellen Kohortenstudie wurden 576 Studenten (medianes Alter knapp 21 Jahre) von 2007 bis 2008 mehrmals kontaktiert und befragt, ob eine Akne bestand, wie sie behandelt wurde, ob in den vergangenen 60 Tagen orale Antibiotika verwendet wurden und ob eine Pharyngitis diagnostiziert worden war. Darüber inspizierten Ärzte auch die Haut und nahmen einen Rachenabstrich vor. Zudem erfolgten Abstriche der Zunge zum Nachweis von Streptococcus salivarius.

358 der Teilnehmer hatten Akne. Von diesen nahmen im Beobachtungszeitraum 36 orale Antibiotika ein (6,2\%), 96 Teilnehmer verwendeten topische Antibioti- ka (16,6\%). Zu den am häufigsten verordneten oral eingenommenen Antibiotika zählten Tetracyclin, Doxycyclin und Minocyclin. Die häufigsten topisch angewendeten Antibiotika waren Clindamycin und Erythromycin.

Die Wahrscheinlichkeit, dass Studienteilnehmer mit oraler Antibiotikatherapie eine Pharyngitis entwickelten, war gegenüber den Teilnehmern ohne diese Behandlung um das 4,34-fache erhöht. Eine Assoziation zwischen topischer Antibiotikatherapie und Pharyngitis ließ sich nicht ableiten.

Bei weniger als 1\% der Studenten waren beta-hämolysierende Streptokokken der Gruppe A in der Mundhöhle nachweisbar. Die Besiedelung mit diesen Keimen zeigte aber keinen Zusammenhang mit einer Pharyngitis.

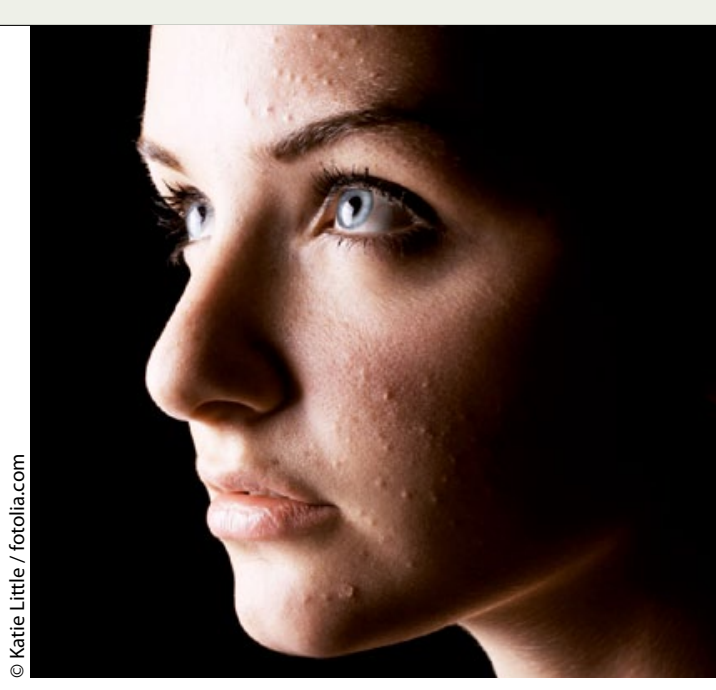

Besser doch nur topische Anwendungen?

Fazit: Es besteht eine enge Assoziation zwischen der Einnahme oraler Antibiotika bei Akne und Pharyngitiden. Ein kausaler Zusammenahng lässt sich aus diesen Daten aber nicht ablesen.

Peter Leiner

Margolis DJ et al. Association of Pharyngitis With Oral Antibiotic Use for the Treatment of Acne. A Cross-sectional and Prospective Cohort Study. Arch Dermatol. 2012; 148 (3): 326-32. doi:10.1001/archdermatol.2011.355

\title{
Akne: Abnehmen, mehr Fisch und weniger Milch?
}

\section{Autoren einer italienischen Multizenterstudie suchten nach verschiedenen Risikofaktoren für mittelschwere und schwere Akne. Interessante Ergebnisse boten sich bei den Ernährungsgewohnheiten.}

\section{$\mathrm{n}$ der Fall-Kontroll-Studie wurden 205 \\ Patienten zwischen zehn und 24 Jahren befragt, bei denen eine mittelschwere oder schwere Akne vorlag. Die Kontrollgruppe bildeten 358 Patienten mit nur leichter bzw. ohne Akne, die aus einem anderen Grund Dermatologen aufgesucht hatten. \\ Genetische Faktoren spielten die Hauptrolle bei der Entwicklung der Akne. Am höchsten war das Risiko der Patienten für eine mittelschwere bzw. schwere Akne,}

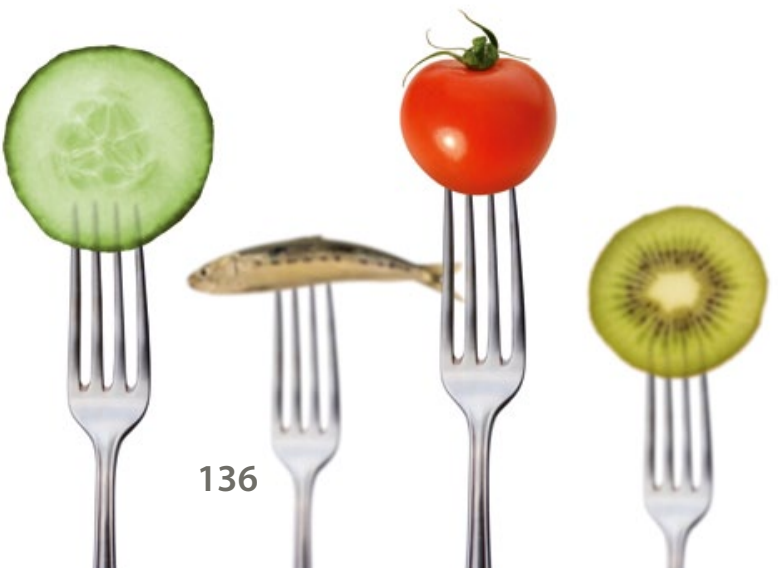

wenn Familienangehörige ersten Grades ebenfalls an der Erkrankung litten (Odds Ratio, OR: 3,41).

Auch bei der Ernährung zeigten sich Auffälligkeiten: Das Aknerisiko war höher, wenn die Jugendlichen mehr als drei Portionen Milch, insbesondere Magermilch, pro Woche zu sich nahmen (OR 1,78). Verglichen mit einem Body Mass Index (BMI)von unter $18,5 \mathrm{~kg} / \mathrm{m}^{2}$ betrug die OR für Akne 1,9, wenn der BMI zwischen 18,5 und $23 \mathrm{~kg} / \mathrm{m}^{2}$ lag und 1,95 bei einem BMI von über $23 \mathrm{~kg} / \mathrm{m}^{2}$. Ein niedriger Body Mass Index dagegen ging mit einem geringeren Aknerisiko einher. Auch wer regelmäßig Fisch auf dem Teller hatte, verzeichnete eine gewisse Schutzwirkung auf seine Haut (OR: $0,68)$.

Anders als in vorausgehenden Studien waren Rauchverhalten oder Menstruati- onsverhalten ohne signifikanten Einfluss. Der Verzehr von Lebensmitteln wie Käse, Joghurt, Brot, Früchten, Gemüse, Fleisch und Wurst ergab ebenfalls keine auffälligen Zusammenhänge. Selbst die viel diskutierten Süßigkeiten, Kuchen und Schokolade trugen nicht zu einer stärkeren Akne bei.

Fazit: Die Daten der aktuellen Studie lassen den protektiven Effekt eines niedrigen BMI vermuten. Die Gesamtstudienlage ist hierzu allerdings noch widersprüchlich. Gleiches gilt für den Einfluss verschiedener Nahrungsmittel auf die Akne. Die ungünstige Wirkung von Magermilch wurde auch in zwei großen amerikanischen Studien beschrieben. Ein interessanter Aspekt ist die offenbar protektive Wirkung von Fisch.

Dr. Christine Starostzik

Di Landro A. et al. Family history, body mass index, selected dietary factors, menstrual history, and risk of moderate to severe acne in adolescents and young adults. J Am Acad Dermatol 2012; Feb 29;

doi:10.1016/j.jaad.2012.02.018 